\title{
Prehabilitation in Paediatric Orthopaedic Surgery Reduces Hospital Length of Stay
}

Clinical Audit

\author{
Mariea A Brady (ID) \\ Holly Carrington' \\ Tim Theologis ${ }^{1,2}$
}

'Medical Sciences Division, Oxford University Hospital, Oxford, UK;

${ }^{2}$ Nuffield Department of Orthopaedics, Rheumatology and Musculoskeletal Science (NDORMS), Oxford, UK
Correspondence: Tim Theologis

NDORMS, Nuffield Orthopaedic Centre,

Windmill Road Headington, Oxford OX3

7HE, UK

Tel +443003047777

Email tim.theologis@ouh.nhs.uk
Purpose: In 2019, the results of the first James Lind Alliance (JLA) research priorities setting partnership (PSP) in paediatric orthopaedics were published in the form of 10 top questions. One of which was, "What is the clinical and cost effectiveness of pre-operative rehabilitation in children presenting with lower limb orthopaedic conditions?" The purpose of this study was to assess the clinical and cost effectiveness of simple pre-operative rehabilitation (prehabilitation) strategies, as measured by hospital Length of Stay (LoS).

Patients and Methods: Clinical records were reviewed retrospectively to determine the average LoS for patients who underwent lower limb paediatric orthopaedic surgery before any prehabilitation intervention. Prehabilitation intervention strategies were introduced, including patient education, provision of crutches and goal setting and subsequent data was collected. LoS before and after intervention were compared. In addition, extra bed days, defined as the difference between actual and expected LoS (determined by the treating clinician with reference to national data), were compared.

Results: Before intervention, the average LoS after paediatric orthopaedic surgery in our hospital was 2.95 days (range 12 days; standard deviation (SD) 2.20 days) and after intervention, the average LoS was reduced to 2.70 days (range 7 days; SD 1.84 days) These data showed an $8 \%$ reduction in hospital LoS after the introduction of prehabilitation. Further, there was a significant $(\mathrm{p}=0.024)$ reduction in extra bed days (per patient) from 1.12 days (range 14 days; SD 1.61 days) before intervention to 0.72 days (range 8 days; SD 1.45 days) after intervention, equating to an estimated saving of $£ 46,500$ in hospital bed costs only.

Conclusion: This data indicates that simple prehabilitation strategies can reduce hospital LoS by $8 \%$ indicating improved clinical outcomes. Further, extra bed days may be reduced by $36 \%$ with potentially important cost savings.

Keywords: lower limb surgery, clinical outcomes, cost effectiveness, recovery after surgery

\section{Introduction}

In 2017, the first James Lind Alliance (JLA) research priorities setting partnership (PSP) in paediatric orthopaedic surgery was established. The JLA is a British non-profit making initiative, which was established in 2004 to bring patients, carers and clinicians together, to identify and prioritise unanswered questions or evidence uncertainties that they agree are the most important. ${ }^{1}$ The aim was to identify and prioritise research questions on the orthopaedic management of lower limb conditions in children. The prioritisation process, including two national surveys of all relevant stakeholders, selected the most important questions producing a list of top-10 research priorities. ${ }^{1}$ One of those was, "What is the clinical and cost effectiveness of pre-operative 
rehabilitation in children presenting with lower limb orthopaedic conditions?" Addressing the priorities established by the JLA PSP is important in establishing evidence-based practice in paediatric orthopaedic surgery, a particularly under-researched and under-funded area.

There is a strong evidence base demonstrating that preoperative strategies to enhance recovery after surgery result in significant improvements in clinical outcomes and cost savings. ${ }^{2}$ However, to date much of this work has focused on the adult surgical population; there is no strong evidence base in the paediatric surgical group. Further, it has been shown that poor outcomes are seen in patients with impaired preoperative functional capacity in the adult population, ${ }^{3}$ but little attention has been given to addressing the preoperative functional capacity of the paediatric surgical population.

Prehabilitation has been described as the practice of enhancing a patient's functional capacity before surgery, with the aim of improving postoperative outcomes., Optimal prehabilitation should be multimodal (eg, medical optimisation, physical exercise, nutritional support, psychological support; such as, is already used in children with complex needs like cerebral palsy) and multidisciplinary (surgeons, physiotherapists, occupational therapists (OT), nurses, etc). In paediatric orthopaedic surgery of the lower limb, simple interventions, such as goal setting with the child and family prior to surgery, are important; this may involve agreeing expected LoS, as well as expected post-operative level of function and pain management. Particularly important in the paediatric surgical group is overcoming psychological barriers such as apprehension when learning a new skill, confidence, and fear of pain. Simple measures such as teaching patients how to use crutches preoperatively and supplying them with crutches prior to surgery may reduce the time taken to return to function, as the child will be comfortable and confident with the supportive devices. Further, prehabilitation may shorten post-operative in-hospital rehabilitation and have a significant impact on hospital LoS.

The aim of this study (registered with our Hospital Research and Development ID 5538) was to determine the LoS of patients undergoing paediatric orthopaedic surgery before and after prehabilitation strategies were implemented. The goal was to introduce prehabilitation strategies that would reduce hospital LoS, as well as improve clinical and cost effectiveness.

\section{Patients and Methods}

The initial step was to review retrospectively clinical records in order to determine the average LoS for patients who underwent paediatric orthopaedic surgery of the lower limb $(n=190)$, prior to any prehabilitation interventions. In this cohort, standard care involved the provision of education and equipment (ie, crutches, frames or slide boards) after surgery, with no interventions before surgery to improve post-operative rehabilitation. After completion of the retrospective review, prehabilitation strategies were introduced and prospective data $(n=134)$ were collected and analysed post intervention. Data were collected as a continuous series for all children aged 5-16 years old undergoing lower limb orthopaedic surgery (consecutive admissions) by any of the 4 paediatric orthopaedic surgeons in our unit. Day cases, bone tumour resections and complex multi-level cerebral palsy surgery were excluded from both the retrospective and prospective cohorts. Retrospective data were collected from 05/01/2016-30/ $12 / 2016$, prospective data were collected from $05 / 10 /$ 2018-27/09/2019. These time frames were selected to ensure that data fully reflected the change in service provision. We avoided including children treated during the transition phase of service provision, as a mixed practice was common place then. We knew for certain that in 2016 there were no pre-habilitation interventions as part of standard care, however, by 2018-2019 prehabilitation interventions were established as standard care in our unit.

Prehabilitation interventions included: (1) education and equipment (eg, crutches, frames or slide boards) provision by physiotherapy and occupational therapy, which were delivered as required prior to surgery; (2) goal setting was agreed between the surgical team and the family in terms of timing and level of function at discharge. These interventions were undertaken in a single session during the children's attendance at the pre-operative assessment clinic and did not lead to extra hospital attendances. Further, all children were directly supervised in their learning of the use of equipment (we did not monitor compliance once they left the clinic). It should be noted that the main difference between standard and prehabilitation protocols was the timing of the interventions. These were delivered pre-operatively in the prehabilitation group and post-operatively in the standard group. It is important to highlight that no additional costs were incurred.

Paediatric lower limb surgery covers a diversity of conditions and operations, nevertheless, there is sufficient 
common ground to consider this sample homogeneous: all children experience lower limb pain and limited joint range of motion after the operation, they need to use walking aids and their hospital stay ranges between 2 and 5 days. As such there was no exclusion criteria, all children undergoing surgery received the same service provision. The study was approved by the Institution's research and development authority (Trust ID 5538), individual consent was not necessary as the collection of data was anonymous and therefore considered as service evaluation.

Expected LoS for each condition/operation was clinician determined prior to the start of data collection and was based on the type and extent of surgery performed, taking into account national data on LoS in paediatric orthopaedic patients (Table 1). ${ }^{6}$ Further, from clinical experience, baseline characteristics of children presenting for lower limb surgery each year is fairly consistent. Clinical practice did not change over the time period evaluated and patients were selected consecutively, both of which would reduce treatment/selection bias.

Finally, an audit discharge proforma, indicating expected LoS, was placed in the front of patients' notes (Figure 1), to capture discharge-related information from the multi-disciplinary team of surgeons, physiotherapists, occupational therapists, nurses, and the pain team.

LoS before and after the intervention were compared. In addition, extra bed days were defined as the difference between actual and expected LoS. The cost of extra bed days per year was calculated as extra bed days multiplied by the estimated average daily cost of a bed, which, according to the Department for Health was approximately $£ 400$ in 2017.

Statistical analysis (independent samples Student's $t$-test to compare before and after intervention group means) was conducted using SPSS (version 25; IBM, New York, New York) with significance set at a $\mathrm{p}<0.05$.

\section{Results}

Prior to the introduction of prehabilitation interventions, the average LoS after paediatric orthopaedic surgery in our hospital was 2.95 days (range 12 days; SD 2.20 days). After the introduction of prehabilitation strategies, the average LoS decreased to 2.70 days (range 7 days; SD 1.84 days). The results showed an $8 \%$ reduction in hospital LoS after prehabilitation (Figure 2). Further, there was a significant $36 \%$ reduction $(\mathrm{p}<0.05)$ in extra bed days (per patient) from 1.12 days (range 14 days; SD 1.61) before intervention to 0.72 days (range 8 days; s.d. 1.45
Table I The Treating Clinician Determined Expected LoS, with Reference to National Data, Following Orthopaedic Surgery of the Lower Limb

\begin{tabular}{|c|c|c|}
\hline Operation & & Days \\
\hline \multicolumn{3}{|l|}{ Foot } \\
\hline \multirow[t]{2}{*}{ Soft Tissue } & Unilateral & 0 \\
\hline & Bilateral & I \\
\hline \multirow[t]{2}{*}{ Bone } & Unilateral & I \\
\hline & Bilateral & 2 \\
\hline \multicolumn{3}{|l|}{ Knee } \\
\hline Scope & & 0 \\
\hline Minor & $\begin{array}{l}\text { 8-plate, patella } \\
\text { reconstruction, } \mathrm{ACL}\end{array}$ & I \\
\hline \multicolumn{3}{|l|}{ Hip } \\
\hline Soft tissue & Uni/Bilateral & I \\
\hline \multirow[t]{3}{*}{$\mathrm{DDH}$} & Closed reduction & I \\
\hline & Open reduction & 2 \\
\hline & OR + Osteotomy & 3 \\
\hline \multirow{2}{*}{$\begin{array}{l}\text { Osteotomies (femur } \pm \\
\text { pelvis) }\end{array}$} & Unilateral & 2 \\
\hline & Bilateral & 3 \\
\hline $\mathrm{CP}$ osteotomies & & 5 \\
\hline \multicolumn{3}{|l|}{ Other } \\
\hline $\begin{array}{l}\text { Lumps/bumps/bone biopsy/ } \\
\text { curettage }\end{array}$ & & 0 \\
\hline Multi-level Surgery & & 7 \\
\hline \multirow[t]{2}{*}{ Ilizarov Frame } & Single & 2 \\
\hline & Complex/Multiple & 3 \\
\hline
\end{tabular}

days) after intervention (Figure 3). This equated to a net total of 117 bed days; with the average LoS per patient being 2.70 days after prehabilitation; an extra 117 bed days would enable 40 more children to be treated. Further, a 36\% reduction in extra bed days resulted in a cost saving on bed cost only of approximately $£ 46,800$ / yr (Table 2).

\section{Discussion}

Paediatric orthopaedic surgery covers an extensive range of musculoskeletal disorders and there is a broad spectrum of interventions, from simple casting through to complex 


\section{Paediatric Orthopaedics \\ Length of Stay Audit}

The Paediatric Orthopaedic team are collecting information on length of stay and discharge arrangements. Please assist us by ensuring this form remains attached to the front of the patient notes and is completed by all the teams involved

\begin{tabular}{|l|l|}
\hline Operation Details: & \\
\hline Operation Date: & \\
\hline Estimated Length of Stay: & \\
\hline Estimated Date of Discharge: & \\
\hline
\end{tabular}

\begin{tabular}{|l|l|l|l|}
\hline \multicolumn{1}{|c|}{ Team } & Involved & Date Discharged & Comments \\
\hline Consultant Team & & & \\
\hline Other Speciality & & & \\
\hline Physiotherapy & & & \\
\hline Occupational Therapy & & & \\
\hline Nursing & & & \\
\hline Pain Team & & & \\
\hline & Required & Date Completed & \\
\hline PoC Arrangements & & & \\
\hline Transport & & & \\
\hline Pharmacy \\
(TrO's pre ordered)
\end{tabular}

Figure I Copy of the audit proforma indicating expected LoS which was placed in the front of patients' notes to be referenced by health care professionals involved in patient care post-surgery.

multi-level surgery. ${ }^{7}$ It is well known that the field is under-researched and under-funded. ${ }^{8-10}$ As a result, the majority of interventions are based on weak evidence.

The James Lind Alliance is funded by the National Institute of Health Research and promotes engagement, communication and discussion between researchers, patients, the public, carers and clinicians to agree on which treatment uncertainties matter most to them and thus set formal future research priorities. The recent James Lind Alliance project on lower limb paediatric orthopaedic surgery has underlined the strong will of children, families and clinicians to address the many unanswered questions in paediatric orthopaedic surgical practice. The ultimate goal is to develop an evidence 


\section{Hospital Length of Stay}

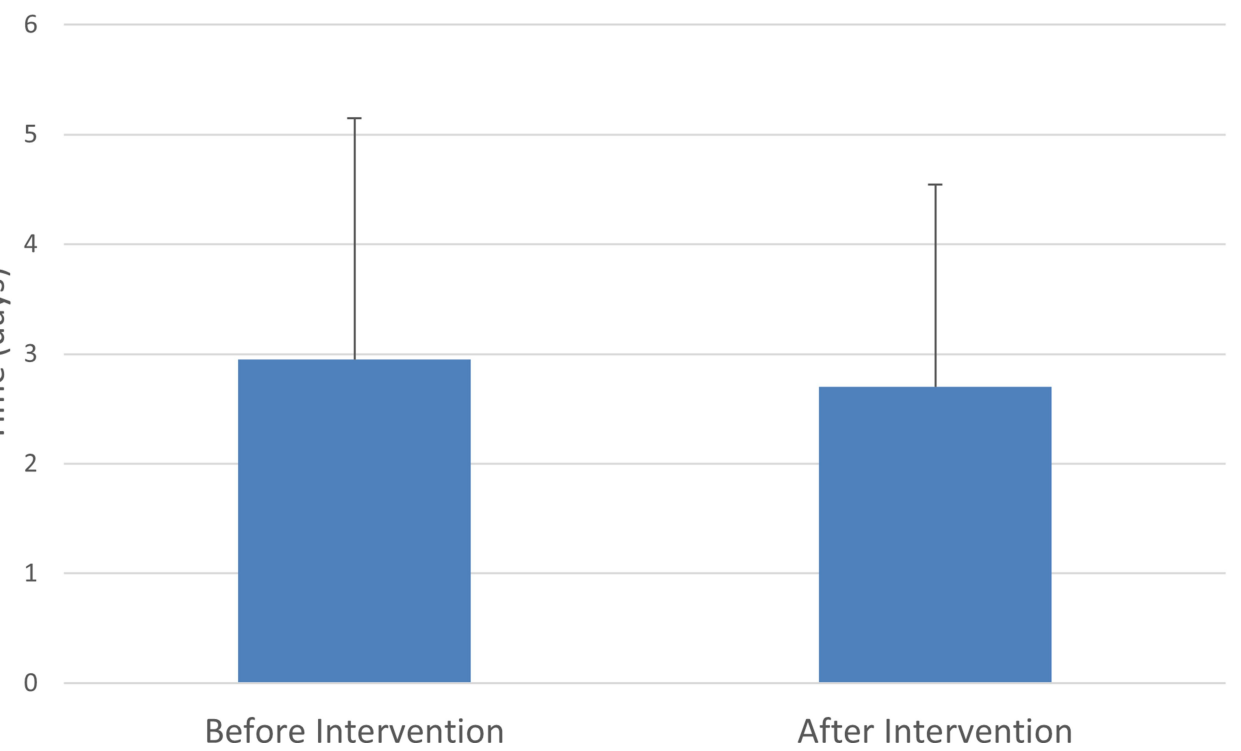

Figure 2 Data before and after the introduction of simple prehabilitation strategies shows a reduction in LoS from 2.95 to 2.70 bed days.

\section{Extra Bed Days (per patient)}

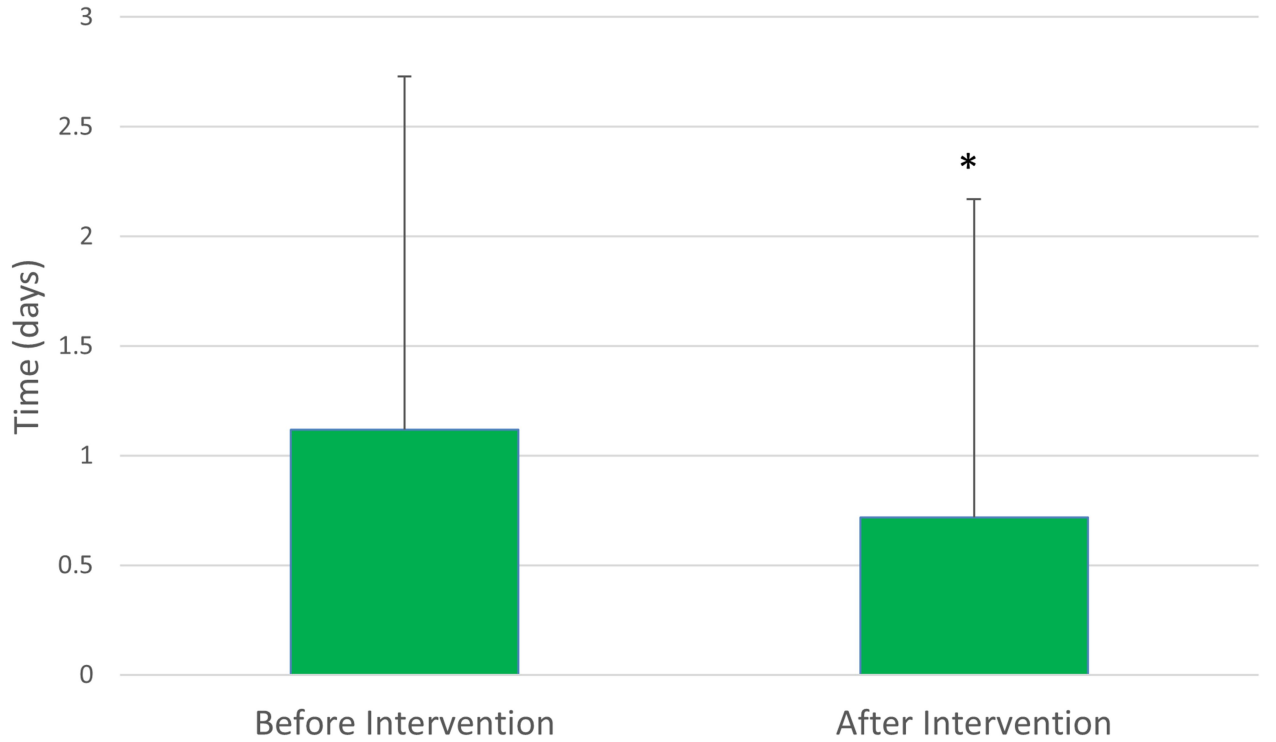

Figure 3 The number of extra bed days per patient was significantly $\left({ }^{*} \mathrm{p}<0.00 \mathrm{I}\right)$ reduced from 1.12 to 0.72 , a decrease of $36 \%$.

base to ensure optimal clinical outcomes, taking into account every stage of the patient journey from presentation in clinic to pre-operative assessment, surgery and post-operative care. The present study aimed at providing preliminary information on one of the highly prioritised questions concerning pre-operative rehabilitation.

Prehabilitation is gaining acceptance as an important factor to enhance recovery after surgery in adult patients. ${ }^{3-5}$
The principle is that improvement of a patient's preoperative functional capacity, both physiologically and psychologically, will improve post-operative clinical outcomes and cost effectiveness. Our literature review indicates that physiological optimisation in the paediatric orthopaedic population has not been formally assessed and it is not known if similar strategies in children are beneficial. It would be logical to assume that pre-operative physiotherapy guided 
Table 2 Cost of Extra Bed Days per Year, Using the Department of Health Estimated Average Daily Cost of a Bed to Be $£ 400$ per Day

\begin{tabular}{|l|c|c|}
\hline Intervention & Extra Bed Days & Cost of Extra Bed Days \\
\hline Before & 213 & $£ 85,200.00$ \\
\hline After & 96 & $£ 38,400.00$ \\
\hline & Saving & $£ 46,800.00$ \\
\hline
\end{tabular}

strength training as well as patient education on the use of auxiliary devices, such as crutches, would enhance postoperative recovery. Further, agreeing on children's expected LoS, level of function and pain management provides welldefined targets, which may contribute to the reduction in LoS.

Hospital LoS is an important metric when evaluating clinical outcomes and cost saving. Shorter LoS would indicate earlier return to function and therefore improved clinical outcome. Similarly, a shorter LoS would reflect a decrease in bed cost per patient. One may extrapolate that with a shorter hospital LoS (indicative of clinical outcome) there would also be a quicker return to school for the child, which would have less impact on education and social isolation from peers. Similarly, the economic impact on parents, who often use annual or unpaid leave to care for their child during hospital stay, would be reduced.

This study has shown for the first time that prehabilitation strategies may decrease hospital LoS following paediatric orthopaedic surgery. After the introduction of simple prehabilitation measures, we observed an $8 \%$ decrease in average hospital LoS. Extra bed days were reduced by $36 \%$ equating to 117 bed days and enabling 40 more children to be treated. Interestingly, a recent systematic review ${ }^{11}$ of the effect of discharge planning from hospital revealed that a personally tailored discharge plan alone led to a small reduction in hospital length of stay ( 0.73 days) as well as a reduction in readmission rates for elderly patients (majority $>75$ years old) with medical conditions. The prehabilitation strategies in this study included a discharge proforma at the front of the patient notes, which indicated expected LoS; this may have contributed to the results. To eliminate this potential bias, future comparative studies must ensure that both the control (standard care) and intervention (prehabilitation) groups have a discharge proforma/planning in place.

From a cost effectiveness perspective, the introduction of prehabilitation strategies in this study added no extra cost. The interventions were delivered at a different time in the patient journey, i.e. pre-operatively. Patients would have normally been taught and provided with crutches after surgery and during their hospital stay. Similarly, the time spent with the physiotherapist, occupational therapist and nurses was moved to pre-operative clinics (rather than during hospital stay post-operatively). Therefore, the prehabilitation strategies added no extra cost and saved $£ 46,800$ per year on bed cost alone. This is a modest estimate, not taking into account other direct or indirect costs, such as in-hospital medications, investigations and interventions or the reduction in parents' requirements for take extra leave from work, travel costs, etc.

Future work will involve design of a prospective randomised control trial, which will include a more extensive prehabilitation intervention. The standardised prehabilitation protocol will be designed in collaboration with physiotherapists to include personalised exercise programmes and patient compliance will be monitored throughout.

\section{Conclusion}

In conclusion, this study demonstrated the potential clinical and cost effectiveness of prehabilitation measures in our settings. Future work will include the design and implementation of a prospective multi-centre comparative clinical trial, which will provide definitive evidence of the clinical and cost effectiveness of pre-operative rehabilitation in children with lower limb orthopaedic conditions.

\section{Acknowledgments}

This study was registered with our Hospital Research and Development (ID 5538) department, which included the required ethics for auditing patient data. This article does not contain any studies with human participants or animals performed by any of the authors.

\section{Author Contributions}

All authors made a significant contribution to the work reported, whether that is in the conception, study design, execution, acquisition of data, analysis and interpretation, or in all these areas; took part in drafting, revising or critically reviewing the article; gave final approval of the version to be published; have agreed on the journal to which the article has been submitted; and agree to be accountable for all aspects of the work.

\section{Disclosure}

The authors report no conflicts of interest in this work. 


\section{References}

1. James Lind Alliance Priority Setting Partnerships. Available from: http://www.jla.nihr.ac.uk/priority-setting-partnerships/Paediatric-lower -limb-surgery. Accessed June 14, 2019.

2. Ljungqvist $\mathrm{O}$, Scott M, Fearon KC. Enhanced recovery after surgery: a review. JAMA Surg. 2017;152(3):292-298. doi:10.1001/ jamasurg.2016.4952

3. Banugo P, Amoako D. Prehabilitation. BJA Educ. 2017;17 (12):401-405. doi:10.1093/bjaed/mkx032

4. Ditmyer MM, Topp R, Pifer M. Prehabilitation in preparation for orthopaedic surgery. Orthop Nurs. 2002;21:43-51. doi:10.1097/ 00006416-200209000-00008

5. Debes C, Aissou M, Beaussier M. Prehabilitation. Preparing patients for surgery to improve functional recovery and reduce postoperative morbidity. Ann Fr Anesth Reanim. 2014;33(1):33-40. doi:10.1016/j. annfar.2013.12.012

6. Civil Eyes Research. Available from: https://www.civil-eyes.com/chil drens/. Accessed June 14, 2019.
7. Gunz AC, Canizares M, Mackay C, et al. Magnitude of impact and healthcare use for musculoskeletal disorders in the paediatric population: a population-based study. BMC Musculoskelet Disord. 2012;13 (98):1-7. doi:10.1186/1471-2474-13-98

8. Dulai SK, Slobogean BL, Beauchamp RD, et al. A quality assessment of randomized clinical trials in pediatric orthopaedics. $J$ Paediatr Orthop. 2007;27(5):573-581. doi:10.1097/bpo.0b013 e3180621f3e

9. Dodwell E, Dua S, Dulai S, et al. The quality of randomized controlled trials in pediatric orthopaedics. J Paediatr Orthop. 2015;35 (5):536-545. doi:10.1097/BPO.0000000000000324

10. Viehweger E, Jouve J, Simeoni M. Outcome evaluation in pediatric orthopedics. Orthop Traumatol Surg Res. 2014;100(1):S113-S123. doi:10.1016/j.otsr.2013.06.012

11. Gonçalves-Bradley DC, Lannin NA, Clemson LM, Cameron ID, Shepperd S. Discharge planning from hospital. Cochrane Database Syst Rev. 2016;(1). doi:10.1002/14651858.CD000313. pub5.

\section{Clinical Audit}

\section{Publish your work in this journal}

Clinical Audit is an international, peer-reviewed, open access journal focusing on the processes and outcomes of clinical audit in any area of healthcare. All aspects of patient care are addressed within the journal and practitioners from all disciplines are invited to submit their work. Areas covered include: Publication of audits; How an audit has changed practice; Practical tips on how to do audits and to

Submit your manuscript here: https://www.dovepress.com/clinical-audit-journal

\section{Dovepress}

avoid pitfalls; How audits have changed patient care; Calls and justifications for new audits. The manuscript management system is completely online and includes a very quick and fair peer-review system, which is all easy to use. Visit http://www.dovepress.com/ testimonials.php to read real quotes from published authors. 